Instituto Internacional de Investigación y Desarrollo Tecnológico Educativo INDTEC, C.A.

DOI: https://doi.org/10.29394/scientific.issn.2542-2987.2016.1.2.3.42-53

OAI-PMH: http://www.indteca.com/ojs/index.php/Revista Scientific/oai

\title{
La Educación Ambiental, como Alternativa para Promover la Convivencia Ecológica
}

\author{
Autora: Betty Marleny Castillo Ávila \\ Universidad Pedagógica Experimental Libertador, UPEL \\ betmarcas@hotmail.com \\ Barinas, Venezuela
}

\section{Resumen}

La presente investigación tiene como propósito Desarrollar un plan de acción en educación ambiental, como alternativa para la convivencia ecológica en los estudiantes de la Escuela Técnica Francisco Tamayo, ubicada en la Parroquia Barinitas, Municipio Bolívar del Estado Barinas. En dicha institución se aprecia que los estudiantes, carecen de una orientación y motivación acerca de un plan de acción para promover la convivencia ecológica. Es una investigación en desarrollo inmersa bajo el enfoque cualitativo, a través del método investigación-acción participante. Su diseño metodológico está estructurado en tres fases: Diagnóstico, programación, conclusiones y propuestas. Los informantes claves estarán representados por tres estudiantes, tres docentes y tres representantes. Las técnicas de recolección de información serán la entrevista en profundidad y la observación de documentos y acciones que muestren construcciones teóricas derivadas de la práctica misma, debido a que se adaptan mejor. Las técnicas de análisis de la información fueron la categorización, triangulación e interpretación. Entre los resultados que se esperan están que los estudiantes se sensibilicen y adquieran conocimientos relacionados con la convivencia ecológica y ambiental; desde un enfoque interdisciplinario, para abordar con responsabilidad los cambios de actitud, que necesita cada comunidad, con la finalidad de desarrollarse social y económicamente.

Palabras clave: educación ambiental; valores; ecología. 


\title{
The Environmental Education, as an Option to Promote the Ecological Coexistence
}

\begin{abstract}
The purpose of this research aims to develop an action plan in environmental education, as an ecologic option for the students of the Francisco Tamayo Technical School located in Barinitas parish, Bolívar municipality state Barinas. In this institution the students lack of an orientation and motivation about an action plan to promote the ecological coexistence. The nature of this study belongs to the qualitative approach of a participatory action research. On the other hand, this research was made in three phases: Phase I. Diagnosis, Phase II Programming, Phase III Conclusions and Proposals. The key people will be: (03) students, (03) teachers and (03) representatives. The technique of gathering information will be the deep interview and documents and actions view which show theoretical structures from the practice itself since so, it represents a better adaptation. The analysis techniques of information were categorization, triangulation and interpretation. Among the expected results are that the students become more sensitive and acquire knowledge related to ecological and environmental cohabitation from an interdisciplinary approach taking responsibility for attitude changes that the community needs to grow socially and economically.
\end{abstract}

Keywords: environmental education; values; ecology.

Date Received: 30-09-2016

Date Acceptance: 17-10-2016 


\section{Introducción}

A escala mundial, la vida diaria del ser humano está rodeada de rápidos y constantes cambios de orden científicos-tecnológicos, que ocasionan diferentes fenómenos que afectan la calidad de vida; tales como el deterioro del ambiente, salud, consumismo, desigualdad, la pobreza extrema y los perjuicios sociales, que ha traído un severo agotamiento de los recursos naturales, contaminación del ambiente y por ende al deterioro general de la sociedad.

Es por eso que, en épocas lejanas, el hombre vivía en equilibrio con el ambiente, poseía una cultura adaptada a las condiciones del entorno, es por ello, que el sistema educativo estaba integrado a garantizar la vida de las generaciones presente en las futuras; de allí, en las últimas décadas gracias al desarrollo tecnológico, tanto a nivel internacional como nacional y local, el hombre ha ido explotando los elementos del sistema natural de forma cada día más acelerada, dejando atrás la cultura ambiental, lo que a menudo ha provocado una sobreexplotación del ambiente.

Es por ello que los estudiantes, deben desarrollar un pensamiento que les permita comprender la problemática ambiental en su conjunto, como una incidencia del sistema en el contexto donde se encuentra, al ser manipulado por la especie humana.

De esta manera, se requiere de un cambio en todo el sistema educativo que va desde la educación inicial hasta la profesional, es así como en las Escuelas Técnicas, demanda de establecer el nivel humano de formación para el desarrollo endógeno por convivencia y manejo del pensamiento complejo; con el propósito de mejorar la conformación del estudiantado, la preparación y el compromiso del profesorado para que, en sus prácticas pedagógicas, se logre la calidad de vida de la población en general. 
Por otra parte, el presente trabajo está estructurado de la siguiente manera:

Momento I: Plantea la contextualización del problema objeto de estudio, se desarrolla el propósito general, específicos y la justificación.

Momento II: Está referido a las perspectivas teóricas, donde se presentan los antecedentes, teorías, enfoques que sustentan la investigación

Momento III: Se plantea el marco metodológico, donde se especifica el tipo de investigación, la metodología, técnicas de recolección de la información a recabar.

\subsection{Visión del Estudio}

\subsubsection{Visión General}

Desarrollar un plan de acción en educación ambiental, como alternativa para la convivencia ecológica en los estudiantes de la Escuela Técnica Francisco Tamayo, ubicada en la Parroquia Barinitas, Municipio Bolívar, Estado Barinas.

\subsubsection{Visión Específica}

Diagnosticar el grado de integración de los estudiantes a las actividades ambientalistas para la convivencia ecológica en los estudiantes de la Escuela Técnica Francisco Tamayo.

Planificar acciones en educación ambiental, como alternativa para la convivencia ecológica en los estudiantes de la Escuela Técnica Francisco Tamayo.

Ejecutar acciones en educación ambiental, como alternativa para la Convivencia Ecológica en los estudiantes de la Escuela Técnica Francisco Tamayo. 
Valorar los resultados de la aplicación de un plan de acción en educación ambiental, como alternativa para la convivencia ecológica en los estudiantes de la Escuela Técnica Francisco Tamayo.

Sistematizar un plan de acción en educación ambiental, como alternativa para la convivencia ecológica en los estudiantes de la Escuela Técnica Francisco Tamayo.

\section{Fundamentación Teórica}

\subsection{Educación Ambiental}

Martínez (2012), "La Educación Ambiental resulta calve para comprender las relaciones existentes entre los sistemas naturales y sociales, así como para conseguir una percepción más clara de la importancia de los factores socioculturales en la génesis de los problemas ambientales (pág. 69). En esta línea se deben impulsar la adquisición de la conciencia, los valores y los comportamientos que favorezcan la participación efectiva de la población en el proceso de toma de decisiones, debe señalarse, que la Educación Ambiental se ha convertido hoy en día en uno de los mejores instrumentos para la correcta orientación de los valores y las conductas humanas para con el ambiente.

\subsection{Convivencia Ecológica}

La educación ambiental basada en el aporte de conocimientos e información que faciliten al hombre interpretar los fenómenos naturales, así como los procesos dinámicos de cambio que ocurren dentro de ellos, es decir que con los conocimientos suministrados por la educación ambiental se pueden explicar los fenómenos climáticos o los ciclos bioquímicos entre otros. Al respecto se hace referencia a la ecología como:

El estudio de la relación entre los seres vivos y su ambiente o de la distribución y abundancia de los seres vivos, y cómo esas 
propiedades son afectadas por la interacción entre los organismos y su ambiente, el ambiente incluye las propiedades físicas que pueden ser descritas como la suma de factores abióticos locales, como el clima y la geología, y los demás organismos que comparten ese hábitat (Salazar y Cajiao, 2012, pág. 158).

En este sentido, la convivencia ecológica permite, al individuo comprender las relaciones de interdependencia con su entorno, a partir del conocimiento reflexivo y crítico de su realidad biofísica, social, política, económica y cultural.

\subsection{Perspectiva Axiológica Ambiental}

Los Valores Ambientales son la dimensión para la consolidación de la conciencia ética - estética y responde al ser, lográndose a través del análisis; así como la reflexión de las propias acciones, al generar en el estudiante el ejercicio de la argumentación como proceso cognitivo, para establecer patrones conductuales favorables. Tal como lo establece el Currículo Básico Nacional (2005), cuando señala:

- Armonía del ambiente, se relaciona con los términos de paz y convivencia, entre el ser humano con todas las especies animales o vegetales que habitan en el planeta.

- Belleza del ambiente, La belleza es un concepto, cualidad abstracta y subjetiva (lo que para unos es bello para otros puede no serlo) presente en la mente de los seres humanos que produce un placer intenso y proviene de manifestaciones sensoriales o ideales.

- Disfrute del ambiente, sensación que brinda el aprovechar algo útil, ventajoso y agradable. Relacionado también con la posesión de algo bueno y agradable, que brinda gozo y placer. Cuando aprovechamos las ventajas del ambiente, su paz, tranquilidad, equilibrio, brillo, color. 
- El deleite hacia el ambiente, al sentir agrado, placer y satisfacción hacia el entorno que nos rodea, esta sensación genera sentimientos de cuido y protección hacia el ambiente.

\section{Acción Metodológica}

\subsection{Naturaleza de la Investigación}

Para dar inicio a la metodología del estudio, el mismo de acuerdo a las sus características se desarrollará en una investigación de paradigma cualitativo. Al respecto se plantea:

La investigación cualitativa se considera como un proceso activo, sistemático y riguroso de indagación dirigida, en el cual se toman decisiones sobre lo investigado. El investigador entra en el campo con una orientación teórica consciente que refleja un conocimiento sustantivo de la teoría de las ciencias sociales. Al mantener un estilo interactivo con el fenómeno objeto de estudio, surge de esa interacción entre ambos las preguntas que orientan la investigación. El investigador y el objeto de investigación se influyen mutuamente. Se ha dicho de ellos que son naturalistas, es decir, que interactúan con los informantes de un modo natural y no intrusivo (Álvarez, 2001 pág. 92).

\subsection{Tipo de Investigación}

El tipo de estudio a utilizar será la Investigación Acción Participativa apoyada en el autor Reza (2000), quien expone que "los objetivos planteados se efectuaran en tres fases; para así facilitar el desarrollo del mismo, siendo necesario seguir las pautas de la investigación acción participativa" (p. 54), en la ejecución de las siguientes pautas:

\section{Primera Etapa. Diagnóstico}

Conocimiento contextual del territorio y acercamiento a la problemática a partir de la documentación existente y de entrevistas a representantes institucionales y asociativos. 


\section{Segunda Etapa. Programación}

Proceso de apertura a todos los conocimientos y puntos de vista existentes, utilizando métodos cualitativos y participativos.

\section{Tercera Etapa. Conclusiones y Propuestas}

Negociación y elaboración de propuestas concretas.

\subsection{Participantes (Informantes Clave)}

Los informantes claves pueden considerarse aquellas personas que cuentan con un conocimiento acerca del medio o problema sobre el que se va a realizar el estudio. Para éste estudio, los informantes claves de la investigación estarán representados por diecisiete (17) estudiantes, diecinueve (19) docente y diecisiete (17) padres y/o representantes seleccionando para ello a tres (3) estudiantes, tres (03) docentes y tres (03) representantes a los cuales se le aplicará una entrevista de profundidad.

\subsection{Técnicas de la Información de la Investigación}

Tamayo y Tamayo (1998), define técnica: "Conjunto de mecanismos, medios y sistemas de dirigir, recolectar, conservar, reelaborar y transmitir los datos y están referidas a la manera como se van a obtener los datos y los instrumentos son los medios materiales" (pág. 59).

Para esta investigación se aplicarán entrevista en profundidad y la observación de documentos y acciones que muestren construcciones teóricas derivadas de la práctica misma, debido a que se adaptan mejor a los objetivos del estudio. La entrevista en profundidad la cual según Taylor y Bogdan (1986), son "reiterados encuentros cara a cara, abiertos no directivos, entre el entrevistador y los informantes, dirigidos hacia la comprensión de las perspectivas que tienen los informantes de su vida, experiencias y situaciones, tal como lo expresan con sus propias palabras" (pág. 107). 


\subsection{Triangulación de la Información}

En cuanto a la triangulación de la información se dice que:

El uso de técnicas de triangulación de los resultados obtenidos durante el trabajo de campo ha gozado de un gran predicamento en la literatura de métodos de investigación social. Una gran parte de los científicos sociales han considerado que cuanto mayor sea la variedad de las metodologías, datos e investigadores empleados en el análisis de un problema específico, mayor será la fiabilidad de los resultados finales (Bisquerra 2000, pág. 167).

En este caso, se considera que la triangulación se efectúa mediante el trabajo realizado directamente en la realidad, basado en hechos sociales que involucran a los sujetos de estudio, al investigador y criterios de posturas teóricas en el desarrollo de un plan de acción en educación ambiental.

\subsection{Categorización}

En cuanto a la categorización, se platea que:

Una buena categorización debe ser tal que exprese con diferentes categorías y precise con propiedades adecuadas lo más valioso y rico de los contenidos protocolares, de tal manera que facilite, luego, el proceso de identificar estructuras y determinar su función, como veremos más adelante, todo lo cual nos encamina con paso firme hacia el hallazgo de teorías o interpretaciones teóricas sólidas y bien fundamentadas en la información protocolar (Martínez 2002, pág. 45).

En este caso, se empleará una metodología basada en descubrir estructuras teóricas no pudiendo consistir en un procedimiento típicamente lineal, sino que sigue básicamente un movimiento en espiral, del todo a las partes y de las partes al todo, aumentando en cada vuelta el nivel de profundidad y de comprensión. 


\section{Resultados y Conclusiones}

Los resultados y conclusiones no se presentan ya que se encuentran en análisis de estudio. A pesar de ello, se muestra una serie de estrategias que se han derivado del análisis de las actividades que hasta ahora se han realizado, que pueden servir de base al momento de la ejecución del plan acción. Entre ellas se encuentran:

- Realizar una videoconferencia sobre los conceptos y alcances sobre la educación ambiental.

- Promover discusiones, talleres, lluvias de ideas, sobre estrategias pedagógicas para la enseñanza de los elementos y conceptos de la educación ambiental dentro de la institución-

- Realizar un mapa conceptual sobre educación ambiental luego de recibir charlas, conferencias y talleres.

- Realizar una caminata ecológica para fomentar el cuido de la naturaleza dentro de la comunidad.

\section{Referencias}

Álvarez, Z., (2001). La investigación cualitativa. Chicago \& Londres: Univiversity of Chicago Press.

Bisquerra, R. (2000). Métodos de Investigación Educativa. Editorial CEAC educación Manuales. Barcelona.

Martínez, M. (2002). Material complementario para la Categorización, Estructuración, Contratación y Teorización en la Investigación Cualitativa. Editorial Morata. Madrid.

Martínez, N, (2012). Enseñanza de la Educación Ambiental. Caracas: Ediciones de la Biblioteca.

Reza, D. (2000). El Proyecto de investigación y sus Aristas. Editorial Colson C.A. Caracas. 
Salazar, M., y Cajiao, L. (2012). La Participación Ciudadana. Madrid: Editorial Popular.

Tamayo y Tamayo, M. (1998). Diccionario de la investigación científica. Limusa. México. 


\section{Castillo Ávila Betty Marleny}

e-mail: betmarcas@hotmail.com

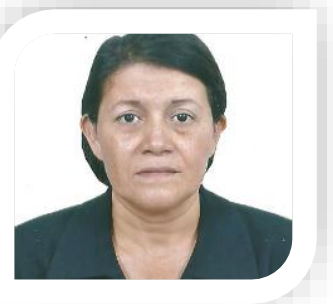

Nacida en Barinitas, Municipio Bolívar del Estado Barinas, residenciada en la Urbanización Mi Futuro, Sector La Carabela, de la Parroquia Barinitas. Graduada en Economía Agrícola, egresada de la Universidad Nacional Experimental de Los Llanos Ezequiel Zamora (UNELLEZ) y en Educación Integral en la Universidad Católica Cecilio Acosta (UNICA)-Maracaibo, año 2010. En la Actualidad laboro como Coordinadora de Extensión en la Escuela Técnica Ambientalista "Francisco Tamayo", ubicada en Barinitas. Cursando estudios de Maestría Educación Ambiente y Desarrollo en la Universidad Pedagógica Experimental Libertador (UPEL) sede Barinas.

El contenido de este manuscrito se difunde bajo una Licencia de Creative Commons ReconocimientoNoComercial-Compartirlgual 4.0 Internacional 